\title{
How Capital Structure And Financial Performance Impact On Food And Beverage Company Value?
}

\author{
Erni Alfisah ${ }^{1}$, Kurniaty $^{2}$, Rizka Zulfikar ${ }^{3 *}$ \\ 1,2,3 Economic Faculty, Islamic University Of Kalimantan MAB \\ Jalan Adhyaksa No. 22 Banjarmasin, Banjarmasin, South Kalimantan, Indonesia \\ ${ }^{*}$ Corresponding author: \\ Email: rizkazulfikar@gmail.com
}

\begin{abstract}
.
The purpose of this study is to examine and explain the effect of capital structure on firm value, the effect of capital structure on financial performance, the effect of financial performance on firm value, the effect of capital structure and financial performance simultaneously on firm value. This study used Food Beverage company that listed in Indonesia Stock Exchange (IDX) on period 2016 - 2020. The model used in data analysis in this study is a simple multiple regression model because the measurement of the dependent and independent variables in this study is in the form of numbers with size scales and more than one independent variable. The results of the study show that: 1) Capital structure has no significant effect on firm value; 2) Capital structure has no significant effect on Financial Performance; 3) Financial Performance has a significant effect on Firm Value; 4) Capital Structure and Financial Performance simultaneously have a significant effect on firm value. The Determination Test (R2) produced is 0.331 (33.1\%), meaning that the contribution of the variable influence of Capital Structure and Financial Performance to Firm Value is $33.1 \%$, while the remaining $66.9 \%$ is influenced by other variables not examined in this study.
\end{abstract}

Keywords: Capital Structure, Financial Performance, Firm Value

\section{INTRODUCTION}

The main goal to be achieved by the company is to maximize shareholder wealth. The purpose is used because by maximizing the value of the company, the owner of the company will be better off or become richer [1]. In practice, these goals are difficult to implement with respect to agency problems. This agency problem arises as a result of the separation of ownership and management of the company. Large companies are usually run by professional managers who do not own or own a small part of the company in question [2]. This separation often leaves managers feeling free, and indeed acting freely in their own best interests, and of course, is often inconsistent with the principle of maximizing shareholder wealth. Management often demands large rewards, in the form of salaries and other facilities.Agency problems come from three main sources, the first source of conflict is the tendency of top managers to ask for various facilities and conditions, not only luxurious facilities, but also sometimes feel entitled to determine various decision strategies [3]. The second source of conflict is the fact that managers often do not own a number of shares in the company [4], so the sense of ownership is reduced, this encourages managers to be too willing to take risks, and do not hesitate to use profits to finance various investment projects. The standard payroll system and limited share ownership make management not optimal in mobilizing energy and attention to maintain the company as the company owner does [5].

Source of conflicts is managers tend to take the safe path, which is too concerned about risk, this results in the loss of investment opportunities that are actually profitable [4].Many factors can affect firm value, but in this study only capital structure variables and financial performance variables are used as factors that can affect 
firm value. Capital structure explains whether changes in funding composition will affect firm value when investment decisions and dividend policy are constant. Within the scope of equilibrium theory, in a perfect capital market and without income tax, capital structure does not affect firm value [6]. The arbitration process will force the value of companies that use debt to be equal to the value of companies that do not use debt. On the other hand, if tax considerations are taken into account, Modigliani and Miller show that the use of debt will always be more profitable than the use of own capital. This is due to the tax-deductible nature of interest payments. Consequently, if the capital market is perfect and there are taxes, then the best capital structure is a capital structure that uses maximum debt [7]. The statement invites controversy, where the controversy actually starts from the assumptions used. It is recognized that if the tax imperfection factor is included, a capital structure that uses as much debt as possible is not an optimal structure.

Likewise, the reluctance of creditors to extend greater credit will make it difficult for companies to work with extreme leverage. Meanwhile, pecking order theory explains why companies have a preference order in choosing funding sources. In accordance with the theory, the company will choose funds that come from operations (internal funds), then only followed by the issuance of bonds that are not risky, issuing risky bonds (such as convertible bonds), and finally issuing new shares [7],[8]. The theory explains why the hierarchy would exist. Hierarchy will occur because of asymmetric information between management and public shareholders.Financial performance is the company's ability to manage and control its resources which will affect the value of the company based on signaling theory about how companies should give signals to the market such as company financial performance information. If a company wants to maximize its value, management must take advantage of the strengths and improve the weaknesses that exist in the company [9]. Financial performance analysis can evaluate the company's financial condition so far and investors who want to buy company shares with a long-term orientation will see the company's ability to generate profits, future prospects and investment risks[10].Capital structure policies can also affect profitability. The initial purpose of the company using debt (leverage) is to meet the lack of funding sources and to increase the company's profitability. If the company does not have a good company performance, then the use of high leverage will reduce the company's profitability [11],[12].

\section{METHOD AND RESEARCH DATA}

The population in this study are food and beverage companies listed on the Indonesia Stock Exchange during the 2016-2020 period. Determination of the sample is carried out using a "non-probability random sampling" approach with a "purposive sampling" method, where the sample selection is based on certain criteria according to the needs and/or information required. The sample criteria are companies that issue financial reports with annual reports, annual financial statements, and ICMD for the 2016-2020 period. The type of data used in this study is secondary data taken from annual reports, annual financial reports, and ICMD of food and beverage companies listed on the Indonesia Stock Exchange for the 2016-2020 period.Financial data was obtained from the Indonesian Capital Market Directory (ICMD), the company's website and the Indonesia Stock Exchange website. This study uses three variables, namely: Capital Structure, Financial Performance and Firm Value. The variables in this study are divided into 2 Independent Variables (Capital Structure and Financial Performance) and one Dependent Variable (Company Value).

The independent variables used in this study are as follows: Capital Structure and Financial Performance as follows:

1. (X1) Capital Structure, using the Debt Assets Ratio (DAR) and Debt to Equity Ratio (DER) indicators

2. (X2) Financial Performance, using Return On Assets (ROA) and Return On Equity (ROE) indicators

The dependent variable used in this study is firm value using 2 indicators, namely Price to Book Ratio (PBV) and Price Earning Ratio (PER).The model used in data analysis in this study is a regression model because the measurement of the dependent and independent variables in this study is a number with a ratio scale measuring 
instrument and the independent variable used is more than one, so that the empirical model used is as follows: following:

$\mathrm{NP}=\beta 0+\beta 1 \mathrm{SM}+\beta 2 \mathrm{KK}$.

Notes :

$\mathrm{NP} \quad=$ Firm Value

$\beta_{0} \quad$ = constant

$\mathrm{SM} \quad=$ Capital Structure

KK $=$ Financial Performance

$\mathrm{B}_{1}-\beta_{2}=$ Regression Coefficient

This research used Fod Beverages company financial performance that listed in Indonesian Stock Exchange (IDX) within 2016-2020 (Table 1.)

Table 1. Food Beverages Company Financial Performance That Listed In IDX Within 2016 -2020.

\begin{tabular}{|c|c|c|c|c|c|c|c|c|c|}
\hline \multirow{2}{*}{ Sil } & \multirow{2}{*}{ Tar } & \multirow{2}{*}{ Canpry Cads } & \multicolumn{7}{|c|}{ BULAMEE BIIIRT } \\
\hline & & & inell & L.tabllise & Equilis & Sat Proll Minr Tas & Tatshar & Choirs: frks & $\mathbf{E s}$ \\
\hline \multirow{5}{*}{1} & 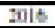 & \multirow{5}{*}{ AEA } & 7.74 .59 & 4.6.7.1.]9 & 43440 & 700. & 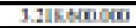 & 1.949 & \begin{tabular}{l|l|l|} 
A. \\
\end{tabular} \\
\hline & ज्ञा7 & & I., 雷 I., & 2.989 .41 & 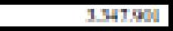 & 3245.413 & 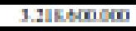 & 492 & 1.494 \\
\hline & 핀 & & TISE+E & $5.29+41$ & 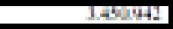 & TDMAा & 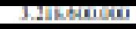 & $7 \mathrm{ps}$ & 75 \\
\hline & II\% & & 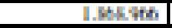 & 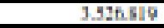 & 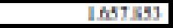 & iㅐ1.34 & 1.115 & IAS & 3990 \\
\hline & III) & & 20M1.535 & T.MUE! & Thents? & 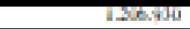 & 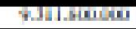 & nat & 2400 \\
\hline & & & & & & & & & \\
\hline \multirow{5}{*}{2} & सा15 & \multirow{5}{*}{ ALTD } & 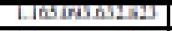 & 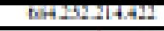 & 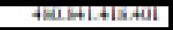 & 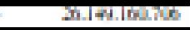 & 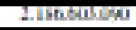 & 30 & 1409 \\
\hline & जाT & & 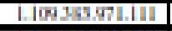 & 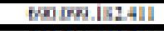 & 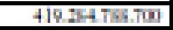 & 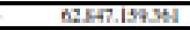 & 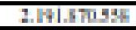 & जIS & IED \\
\hline & Dint: & & TWHASHAT & 7LTH & 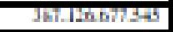 & 24.15.11.155 & Ifrithus:- & $+\infty$ & 130 \\
\hline & 细神 & & 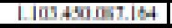 & T21.T19.9h1.450 & 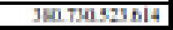 & 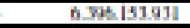 & 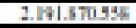 & 焉 & 3.16 \\
\hline & III) & & 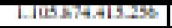 & 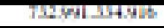 & 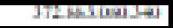 & 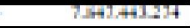 & 2.7. & now & 4, 嘈 \\
\hline & & & & & & & & & \\
\hline \multirow{5}{*}{1} & 민해 & \multirow{5}{*}{ BLDI } & 2.711.1201 & innthenes & 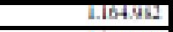 & 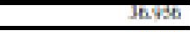 & 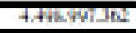 & WI & 7 \\
\hline & $\$ 17$ & & 2.71445 & 1.744 .79 & 1.19470 & $4 \pi+4$ & 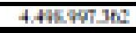 & 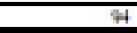 & P.1] \\
\hline & 到16 & & 1.34440 & 2. Inteftite & $112 \mathrm{x}+4$ & 4 도핌 & 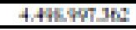 & 常 & 吅带 \\
\hline & פiा? & & 1.24.967 & 1.914 .479 & 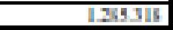 & 10.990 & 4. Ahenthes & 19 & 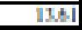 \\
\hline & III) & & 2.7voum & 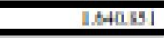 & 1.12215 & thess & 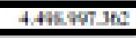 & क & 13.5. \\
\hline \multirow{5}{*}{4} & 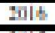 & \multirow{5}{*}{$\mathrm{CER}$} & 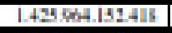 & 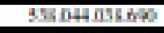 & 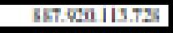 & 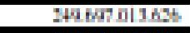 & 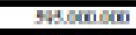 & 195 & $4+29$ \\
\hline & त्या? & & 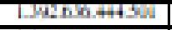 & 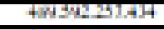 & 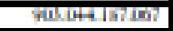 & 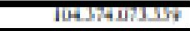 & 5करणनाIIIII & 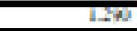 & IIII \\
\hline & 핀 & & 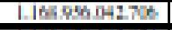 & 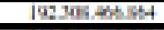 & 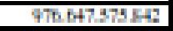 & I017.7. & 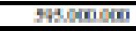 & 11579 & 115 \\
\hline & Int? & & 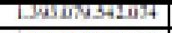 & 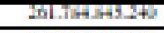 & 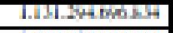 & 11-17.1Bum & FHstrim & inter & 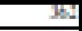 \\
\hline & 11130 & & 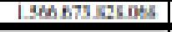 & H40.95.51134 & $1.961 .71-69 \mathrm{FHH}_{4}$ & 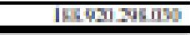 & 5HAgming & 11.75 & 19: \\
\hline \multirow{5}{*}{9} & 피늘 & \multirow{5}{*}{ DLTA } & 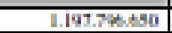 & 199.411.44 & 1.012.274 & 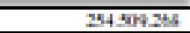 & 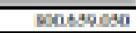 & $50 \mathrm{~s}$ & 117 \\
\hline & IIII & & 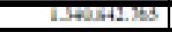 & 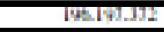 & TITIDIM! & Whandit & 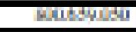 & $43+4$ & 팩 \\
\hline & 国1 & & 14215117.170 & 고고.191.196 & 1.21H.169.14 & 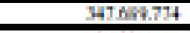 & 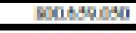 & 250 & 411 \\
\hline & 에과 & & 1.19.061.712 & 기1243.140 & 1.213 .0697112 & 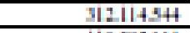 & mondapdi & 5n & 391 \\
\hline & \$II & & 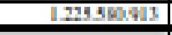 & 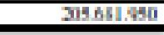 & 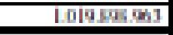 & 115.918h\| & 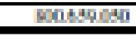 & 4.40 & 119 \\
\hline \multirow{5}{*}{$t$} & 斯贵 & \multirow{5}{*}{$10 \mathrm{~T}$} & 25.61144t & 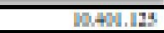 & | & 3 319.91E & 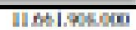 & 547 & 19: \\
\hline & \$인 & & 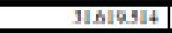 & 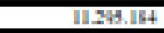 & 5454515 & 1.111.290 & 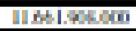 & 165 & 197 \\
\hline & IIII & & Howing & Ifring & 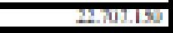 & 5Brifit & 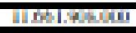 & 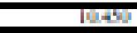 & Thy \\
\hline & 피간 & & 14.720.114 & 11.014 .210 & shintint & 9.72459 & 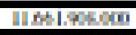 & 11.15 & 419 \\
\hline & 11150 & & ImIsting & 5156191 & 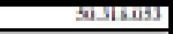 & 9.411. & 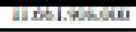 & 7315 & 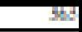 \\
\hline & & & & & & & & & \\
\hline & II5 & & 2.124.515 & 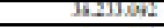 & 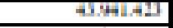 & $535 \times$ & 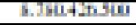 & 2025 & FIII \\
\hline & 1911 & & FH.4AIIT) & 41.3\%.|l|| & 47.10274 & 4.541.2419 & 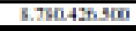 & Th19 & 477 \\
\hline 7 & IIt & MTH & 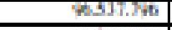 & +thanth & 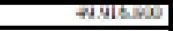 & 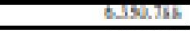 & 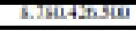 & 1,50 & 717 \\
\hline & 교단 & & 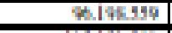 & 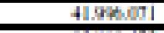 & 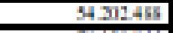 & Esfind & B.750425.901 & 7929 & 959 \\
\hline & III & & 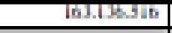 & DWhat & B.D. & 9241.115 & 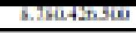 & thes & III \\
\hline & & & & & & & & & \\
\hline & 페늘 & & minsulat & Bataling & Nownts & $425.5 \%$ & 1. & $1.75=$ & 7 里 \\
\hline ह & 2011 & & $2.210 \mathrm{~m}$ & 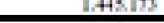 & $10 \mathrm{n}+\mathrm{h}$ & 1sont & 2.107nangin & 11 kis & 첼1 \\
\hline & InI & MILBI & 1.840.901 & $1.711 .7 \mathrm{a}$ & 1.167515 & 1.293 .44 & 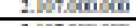 & thimb & 251 \\
\hline & IIIf & & Antaq9 & $1,154+2$ & $1.1+5000$ & $130 \pi, 4$ & 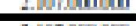 & 19 aps & 297 \\
\hline & $\ldots$ & & Intits & $1,4+460$ & $1,4 \sqrt{1}+\infty$ & 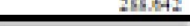 & 1.whing in & $\operatorname{mon}$ & ए1] \\
\hline & 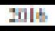 & & 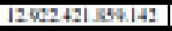 & 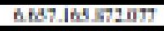 & 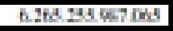 & 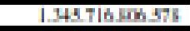 & 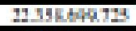 & IBHE & 빌 \\
\hline & 핀 & & 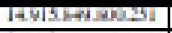 & 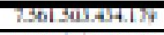 & 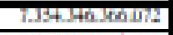 & 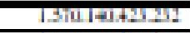 & Musterns & $2 \pi y$ & II \\
\hline 9 & 피는 & Mnds & 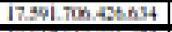 & 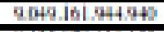 & 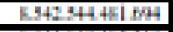 & 1.104.74.191.197 & 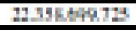 & 7 ans & 77 \\
\hline & IIIt? & & 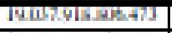 & 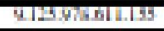 & 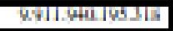 & 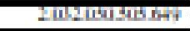 & 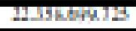 & 21150 & 잠닥 \\
\hline & "113: & & 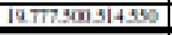 & [1. & 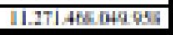 & 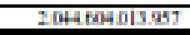 & 1199064793 & 175 & 翌 \\
\hline & 니낸 & & 6.54 & 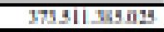 & 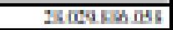 & $41 \mathrm{Wh} .41 .761$ & I. + tovenen & 14 & .1214 \\
\hline & Iजा? & & 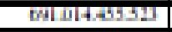 & 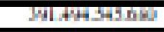 & 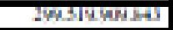 & 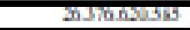 & 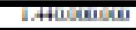 & 25 & Th, \\
\hline in & 牙h & Fुप4 & 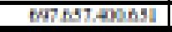 & 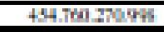 & 14:mismbin & 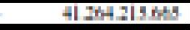 & 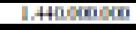 & 17 & 48.7 \\
\hline & 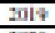 & & 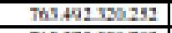 & angangur.44 & 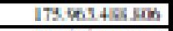 & ㄹ. 372 . 11.712 & 1.4toumang & [15] & -1125 \\
\hline & \$II) & & 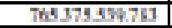 & 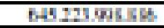 & 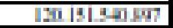 & 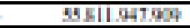 & 1. 4 Hornom & $1 \mathrm{D}$ & $4 \mathrm{MO}$ \\
\hline
\end{tabular}




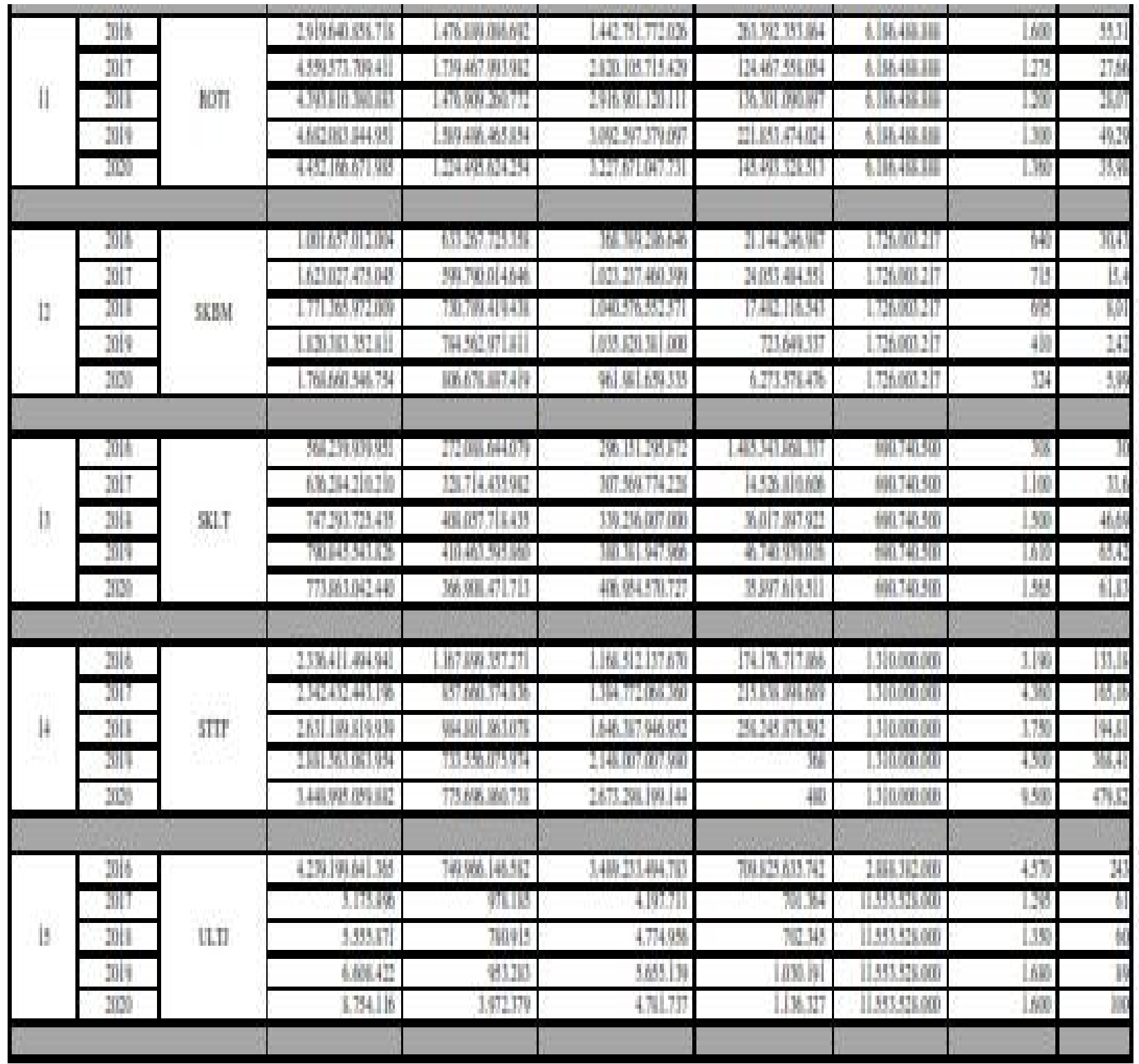

\section{RESULT AND DISCUSSION}

Our result study such as $\mathrm{t}$ and $\mathrm{F}$ Test; multiple regression analysis and ANOVA are presented in Table 2 -6 below.

Table 2. T Test Between Capital Stucture and Company Value

Coefficients $^{\mathrm{a}}$

\begin{tabular}{|c|c|c|c|c|c|}
\hline & \multicolumn{2}{|c|}{ Unstandardized Coefficients } & \multirow{2}{*}{\begin{tabular}{|l}
$\begin{array}{l}\text { Standardized } \\
\text { Coefficients }\end{array}$ \\
Beta \\
\end{tabular}} & \multirow[b]{2}{*}{$\mathrm{T}$} & \multirow[b]{2}{*}{ Sig. } \\
\hline Model & $\mathrm{B}$ & Std. Error & & & \\
\hline (Constant) & 1050865.250 & 523222.443 & & 2.008 & .048 \\
\hline Capital Structure & 181524.438 & 451322.123 & .047 & .402 & .689 \\
\hline
\end{tabular}

a. Dependent Variable: Company Value 
Single Regression Equation is :

$\mathrm{Y}=1050865.250+181524.438 \mathrm{X}+\mathrm{e}$

Where :

$$
\begin{aligned}
& \mathrm{Y}=\text { Company Value } \\
& \mathrm{X}=\text { Capital Structure } \\
& \mathrm{e}=\text { Standard error }
\end{aligned}
$$

\begin{tabular}{|c|c|c|c|c|c|}
\hline \multirow[b]{2}{*}{ Model } & \multicolumn{2}{|c|}{ Unstandardized Coefficients } & \multirow{2}{*}{\begin{tabular}{|l} 
Standardized \\
Coefficients \\
Beta \\
\end{tabular}} & \multirow[b]{2}{*}{$\mathrm{t}$} & \multirow[b]{2}{*}{ Sig. } \\
\hline & B & Std. Error & & & \\
\hline $1 \quad$ (Constant) & .253 & .076 & & 3.314 & .001 \\
\hline Capital Sturcture & -.118 & .066 & -.204 & -1.784 & .079 \\
\hline
\end{tabular}

Table 3. $T$ Test Between Capital Structure and Financial Performance

a. Dependent Variable: Financial Performance

Single Regression Equation is :

$\mathrm{Y}=0.253-0.118 \mathrm{X}+\mathrm{e}$

Where :

$$
\begin{aligned}
& \mathrm{Y}=\text { Financial Performance } \\
& \mathrm{X}=\text { Capital Structure } \\
& \mathrm{e}=\text { Standard error }
\end{aligned}
$$

\begin{tabular}{|c|c|c|c|c|c|}
\hline \multirow[b]{2}{*}{ Model } & \multicolumn{2}{|c|}{ Unstandardized Coefficients } & \multirow{2}{*}{\begin{tabular}{|l} 
Standardized \\
Coefficients
\end{tabular}} & \multirow[b]{2}{*}{$\mathrm{t}$} & \multirow[b]{2}{*}{ Sig. } \\
\hline & B & Std. Error & & & \\
\hline $1 \quad$ (Constant) & 856691.795 & 385678.281 & & 2.221 & .029 \\
\hline $\begin{array}{l}\text { Financial } \\
\text { Performance }\end{array}$ & 2089577.137 & 745792.403 & .312 & 2.802 & .007 \\
\hline
\end{tabular}

Table 4. T Test Between Capital Structure and Financial Performance

\section{Coefficients $^{\mathrm{a}}$}

a. Dependent Variable: Company Value

Single Regression Equation is :

\begin{tabular}{|c|c|c|c|c|c|}
\hline \multirow[b]{2}{*}{ Model } & & Coefficients & $\begin{array}{l}\text { Standardized } \\
\text { Coefficients }\end{array}$ & & \multirow[b]{2}{*}{ Sig. } \\
\hline & B & Std. Error & Beta & $\mathrm{t}$ & \\
\hline
\end{tabular}

$\mathrm{Y}=856691.795+2089577.137 \mathrm{X}+\varepsilon$

Where ::

$$
\begin{aligned}
& \mathrm{Y}=\text { Company Value } \\
& \mathrm{X}=\text { Financial Performance } \\
& \mathrm{e}=\text { Standard error }
\end{aligned}
$$

Table 5. Multiple Regression Analysis Result

\section{Coefficients ${ }^{\mathrm{a}}$}




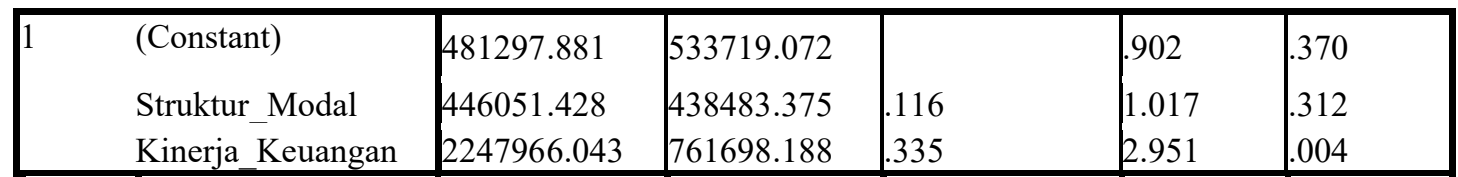

a. Dependent Variable: Company Value

Based on the table 5 above, it can be formed multiple linear regression equation for this study as follows:

$\mathrm{Y}=481297.881+446051.428 \mathrm{X}_{1}+2247966.043 \mathrm{X}_{2}+\varepsilon$

where :

$\mathrm{Y} \quad=$ Firm value

$\mathrm{X}_{1} \quad=$ Capital Structure

$\mathrm{X}_{2} \quad=$ Financial Performance

$\varepsilon \quad=$ Standard error

Table 6. Simultaneous Analysis

ANOVA $^{\mathrm{a}}$

\begin{tabular}{|ll|l|l|l|l|l|}
\hline Model & & Sum of Squares & Df & Mean Square & F & Sig. \\
\hline 1 & Regression & 8950164029396 & & 4475082014698 & 4.444 & $.015^{\mathrm{b}}$ \\
& & 4.770 & & 2.380 & & \\
& & & & \\
Residual & 7249734258415 & 72 & 1006907535891 & & \\
Total & 37.000 & 0.236 & & \\
& 8144750661355 & 74 & & & \\
\hline
\end{tabular}

a. Dependent Variable: Company Value

b. Predictors: (Constant), Financial Performance, Capital Structure

Hypothesis 1 which shows that capital structure has a significant effect on firm value is rejected with a significance value of 0.689 and greater than 0.05 . The direction of the influence of capital structure on firm value is positive, which means that the larger the capital structure, the greater the firm value. This result is in accordance with the MM theory which states that an increase in debt can increase the value of the company if it has not reached its optimal point, this is reinforced by the trade-off theory which explains that the use of debt can reduce the tax burden. and corporate agency costs [13],[14],[15].

Hypothesis 2 which states that capital structure has a significant effect on financial performance is rejected with a significance value of 0.079 and greater than 0.05 . The direction of the influence of capital structure on financial performance is negative, which means that the greater the debt-based capital structure, the lower the financial performance. This result is in accordance with the view expressed which states that the larger the debt-based capital structure as a burden on the company, the lower the financial performance. And the declining level of profitability is caused by the costs that must be borne by the company when they use a high level of debt [16], [17], [18].

Hypothesis 3 which states that financial performance has a significant effect on firm value is accepted with the significance value of financial performance being 0.007 and less than 0.05 . The direction of the influence of financial performance on firm value is positive, which means the better the financial performance, the higher the firm value. These results are in accordance with the theory and previous research that became the basis for the formulation of the hypothesis.

The right theory to relate the influence of financial performance to firm value is signaling theory about how companies should signal to users of reports in the form of information about the company's financial performance [19]. There is a less expensive method for companies to signal to investors that the company can 
issue announcements about the company's prospects and ability to generate profits by hiring outsiders to examine the company's books or other materials and provide an opinion on whether managers are telling the truth [20], [21].

If company want to maximize the value of the company, management must take advantage of existing strengths and improve existing weaknesses in the company [22]. An investor will buy company shares with a long-term orientation to see the company's ability to generate profits, future prospects, and investment risks in the company, thus a company that has good financial performance is a good signal in increasing the value of the company. company. The results of this study support several previous studies. The effect of financial performance on firm value has been empirically proven by several researchers. These studies results show that financial performance has a positive effect on firm value [23], [24]. [25] whose results show that financial performance as measured by using the ROA indicator has a significant effect on firm value using the Tobin's Q, PER, and closing price indicators. [10] research results show that financial performance with indicators of ROA and $\mathrm{ROE}$ has a positive effect on firm value.

Hypothesis 4 which states that capital structure and financial performance have a significant effect on firm value is accepted with the significance value of financial performance being 0.015 and less than 0.05 . This result is in accordance with the MM theory which states that an increase in debt (capital structure) can increase firm value if it has not reached its optimal point, this is reinforced by the trade-off theory which explains that the use of debt can reduce the company's tax burden and agency costs [24]. The right theory to relate the influence of financial performance to firm value is signaling theory about how companies should signal to report users in the form of information about the company's financial performance.

There is a less expensive method for companies to signal to investors that the company can issue announcements about the company's prospects and ability to generate profits by hiring outsiders to examine the company's books or other materials and provide an opinion on whether managers are telling the truth [16], [22]. .If commpny want to maximize the value of the company, management must take advantage of existing strengths and improve existing weaknesses in the company. An investor will buy company shares with a long-term orientation to see the company's ability to generate profits, future prospects, and investment risks in the company, thus a company that has good financial performance is a good signal in increasing the value of the company [12],[23].

\section{CONCLUSION}

Financial performance has a significant effect on firm value in a positive direction, meaning that the greater the financial performance, the greater the firm value. These results confirm the signaling theory about how companies should signal to users of reports in the form of information about the company's financial performance. There is a cheaper method for companies to signal to investors that the company can issue announcements about the company's prospects and ability to generate profits by hiring outsiders to examine the company's books or other materials and provide an opinion on whether managers are telling the truth.

Capital structure and financial performance simultaneously have a significant effect on firm value. These results confirm the MM theory which states that an increase in debt (capital structure) can increase firm value if it has not reached its optimal point, this is reinforced by the trade-off theory which explains that the use of debt can reduce the company's tax burden and agency costs. While the right theory to link the effect of financial performance on firm value is signaling theory about how companies should signal to users of reports in the form of information about the company's financial performance. Based on the results of the determination, it can be seen that the adjusted R2 produced is 0.331 , meaning that the percentage contribution of the influence of the capital structure and financial performance variables on the firm value is $33.1 \%$, while the remaining $69.9 \%$ is influenced by other variables not included in this model. 


\section{REFERENCE}

[1] Vătavu, S. (2015). The impact of capital structure on financial performance in Romanian listed companies. Procedia Economics and Finance, 32, 1314-1322.

[2] Dang, H. N., Vu, V. T. T., Ngo, X. T., \& Hoang, H. T. V. (2019). Study the impact of growth, firm size, capital structure, and profitability on enterprise value: Evidence of enterprises in Vietnam. Journal of Corporate Accounting \& Finance, 30(1), 144-160.

[3] Abdullah, H., \& Tursoy, T. (2021). Capital structure and firm performance: evidence of Germany under IFRS adoption. Review of Managerial Science, 15(2), 379-398.

[4] Ayo-Oyebiyi, G. T. (2019). Capital Structure and Organizational Performance: Evidence from Nigerian Food and Beverage Companies. South Asian Journal of Social Studies and Economics, 1-9.

[5] Das, C. P., \& Swain, R. K. (2018). Influence of capital structure on financial performance. Parikalpana: KIIT Journal of Management, 14(1), 161-171.

[6] Santoso, H., Lako, A., \& Rustam, M. (2020). Relationship of Asset Structure, Capital Structure, Asset Productivity, Operating Activities and Their Impact on the Value of Manufacturing Companies Listed on the Indonesia Stock Exchange. International Journal of Multicultural and Multireligious Understanding, 7(8), 358-370.

[7] Ramli, N. A., Latan, H., \& Solovida, G. T. (2019). Determinants of capital structure and firm financial performance-A PLS-SEM approach: Evidence from Malaysia and Indonesia. The Quarterly Review of Economics and Finance, 71, 148-160.

[8] Antoro, W., Sanusi, A., \& Asih, P. (2020). The Effect of Profitability, Company Size, Company Growth on Firm Value Through Capital Structure in Food and Beverage Companies on the Indonesia Stock Exchange 2014-2018 Period. International Journal of Advances in Scientific Research and Engineering, 6(09), 36-43.

[9] Desai, J., \& Desai, R. (2019). Capital Structure as Determinant of Financial Performance: Review of Literature. Copernican Journal of Finance \& Accounting, 8(4), 133-148.

[10] Dinova, R. D. K., \& Herawati, A. (2019). The Effect Of Profitability, Capital Structure, and Corporate Value On Stock Return (Pharmaceutical Sub Sector In Indonesia Stock Exchange 2015-2017). Dinasti International Journal of Education Management And Social Science, 1(2), 224-234.

[11] Suhadak, S., Kurniaty, K., Handayani, S. R., \& Rahayu, S. M. (2019). Stock return and financial performance as moderation variable in influence of good corporate governance towards corporate value. Asian Journal of Accounting Research.

[11] Kusumawati, R., \& Irham, R. (2018, April). The Effect of Capital Structure and Profitability to Firm Value with Managerial Ownership as a Variable Moderation. In GLOBAL MULTIDISCIPLINARY RESEARCH CONFERENCE $(G M R C)$ (p. 14).

[12] Maryam, D., \& Ramadhani, Y. C. (2019). Agency Cost as An Intervening Variable in the Impact of Capital Structure and Company Size on Company Performance. Jurnal Akuntansi, Manajemen dan Ekonomi, 21(3), 54-60.

[13] Akbar, M. N., \& Rahma, M. (2022). THE DETERMINING FACTORS OF CORPORATE VALUE: EMPIRICAL STUDY OF FOOD AND BEVERAGE COMPANIES LISTED ON THE INDONESIA STOCK EXCHANGE. Academy of Accounting and Financial Studies Journal, 26, 1-11.

[14] Setiyowati, S. W., Naser, J. A., \& Astuti, R. (2020). Leverage dan growth opportunity mempengaruhi nilai perusahaan melalui profitabilitas. Jurnal Ekonomi Modernisasi, 16(1), 31-40.

[15] Dang, T. D., \& Do, T. V. T. (2021). DOES CAPITAL STRuCTuRE AFFECT FIRM VALuE IN VIETNAM?. Investment Management and Financial Innovations, 18(1), 33-41.

[16] Phuong, N. T. T., \& Hung, D. N. (2020). Impact Of corporate governance on corporate value: Research in Vietnam. Research in World Economy, 11(1), 161-170.

[17] NGUYEN, L., TAN, T. K. P., \& NGUYEN, T. H. (2021). Determinants of Firm Value: An Empirical Study of Listed Trading Companies in Vietnam. The Journal of Asian Finance, Economics and Business, 8(6), 809-817.

[18] SUDIYATNO, B., PUSPITASARI, E., SUWARTI, T., \& ASYIF, M. M. (2020). Determinants of firm value and profitability: Evidence from Indonesia. The Journal of Asian Finance, Economics, and Business, 7(11), 769-778.

[19] NGUYEN, L., TAN, T. K. P., \& NGUYEN, T. H. (2021). Determinants of Firm Value: An Empirical Study of Listed Trading Companies in Vietnam. The Journal of Asian Finance, Economics and Business, 8(6), 809-817.

[19] VENUSITA, L., \& AGUSTIA, D. (2021). The Relationship Between Firm Value and Ownership of Family Firms: A Case Study in Indonesia. The Journal of Asian Finance, Economics and Business, 8(4), 863-873.

http://ijstm.inarah.co.id 
[20] SUDIYATNO, B., PUSPITASARI, E., SUWARTI, T., \& ASYIF, M. M. (2020). Determinants of firm value and profitability: Evidence from Indonesia. The Journal of Asian Finance, Economics, and Business, 7(11), 769-778.

[21] Lee, S. M., \& Ryu, K. (2020). Management Ownership and Firm Value: An Empirical Analysis using Panel Data. Seoul Journal of Economics, 33(1).

[22] Mahzura, T. A. S. (2018). The Analysis of The Influence of Financial Performance, Company Size, Ownership Structure, Leverage and Company Growth on Company Values in Food and Beverage Industry Companies Listed in IDX 2012-2016 Period. International Journal of Public Budgeting, Accounting and Finance, 1(4), 1-12.

[23] Cahyaningtyas, N. W., \& Muharam, H. (2022). DETERMINANTS OF CAPITAL STRUCTURE AND FIRM FINANCIAL PERFORMANCE: EMPIRICAL EVIDENCE ON FOOD AND BEVERAGE SECTOR IN INDONESIAN LISTED COMPANIES. Academy of Accounting and Financial Studies Journal, 26, 1-10. 